\title{
BACKWARD PERTURBATION ANALYSIS OF THE PERIODIC DISCRETE-TIME ALGEBRAIC RICCATI EQUATION*
}

\author{
JI-GUANG $\mathrm{SUN}^{\dagger}$
}

\begin{abstract}
Normwise backward errors and residual bounds for an approximate Hermitian positive semidefinite solution set to the periodic discrete-time algebraic Riccati equation are obtained. The results are illustrated by using simple numerical examples.
\end{abstract}

Key words. periodic Riccati equation, Hermitian positive semidefinite stabilizing solution set, backward error, residual bound

AMS subject classifications. 15A24, 65H10, 93B99

DOI. $10.1137 / \mathrm{S} 0895479802414928$

1. Introduction. We consider the periodic discrete-time algebraic Riccati equation (P-DARE) with period $p \geq 2$ :

$$
\begin{aligned}
X_{j-1} & =A_{j}^{H} X_{j} A_{j}-A_{j}^{H} X_{j} B_{j}\left(R_{j}+B_{j}^{H} X_{j} B_{j}\right)^{-1} B_{j}^{H} X_{j} A_{j}+H_{j} \\
& =A_{j}^{H} X_{j}\left(I+G_{j} X_{j}\right)^{-1} A_{j}+H_{j},
\end{aligned}
$$

where, for all $j, A_{j}=A_{j+p}, H_{j}=H_{j+p}$ and $X_{j}=X_{j+p}$ are $n \times n$ matrices, $B_{j}=B_{j+p}$ are $n \times m$ matrices, and $R_{j}=R_{j+p}$ are $m \times m$ matrices; $B_{j}$ is of full column rank, $R_{j}$ is Hermitian positive definite $\left(R_{j}>0\right), G_{j}=B_{j} R_{j}^{-1} B_{j}^{H}=G_{j+p}$, and $H_{j}$ is Hermitian positive semidefinite (p.s.d.) with $H_{j}=C_{j}^{H} C_{j}$. Equation (1.1) arises frequently in solving periodic discrete-time linear optimal control problems [1], [2]. Appropriate assumptions on the coefficient matrices guarantee the existence and uniqueness of the Hermitian p.s.d. stabilizing solution set $\left\{X_{j}\right\}_{j=1}^{p}$ to the P-DARE (1.1) (see Theorem 2.5 of section 2 ). Note that for the case $p=1$ we have a single Riccati equation for which backward perturbation bounds and residual bounds are known [15], [16].

A forward perturbation analysis of the P-DARE (1.1) is presented by Lin and Sun [12], where perturbation bounds and condition numbers of the Hermitian p.s.d. stabilizing solution set to the P-DARE are obtained [12, sections 3 and 4]. In this paper, we present a backward perturbation analysis of the P-DARE (1.1).

Backward perturbation analysis is motivated by the following fact. Let an approximate Hermitian p.s.d. solution set $\left\{\tilde{X}_{j}\right\}_{j=1}^{p}$ to the P-DARE (1.1) be given. For example, the approximate solution set may come from a numerical algorithm for approximating the exact Hermitian p.s.d. stabilizing solution set $\left\{X_{j}\right\}_{j=1}^{p}$. Then there are two questions associated with the approximate solution set: (1) Is the approximate solution set the exact solution set of a slightly perturbed P-DARE? (2) Is the approximate solution set close to the exact solution set $\left\{X_{j}\right\}_{j=1}^{p}$ ? The result of a backward perturbation analysis may be a backward error, or a residual bound. The purpose of backward perturbation analysis of the P-DARE (1.1) is to test the stability

*Received by the editors September 23, 2002; accepted for publication (in revised form) by V. Mehrmann July 22, 2003; published electronically August 6, 2004. This work was supported by the Swedish Strategic Research Foundation Grant entitled "Matrix Pencil Computations in ComputerAided Control System Design: Theory, Algorithms and Software Tools."

http://www.siam.org/journals/simax/26-1/41492.html

${ }^{\dagger}$ Department of Computing Science, Umeå University, S-901 87 Umeå, Sweden (jisun@cs.umu.se). 
of a computation or an algorithm and to ascertain the accuracy of an approximate solution set.

In matrix computations, developing backward errors and residual bounds is a part of the subject of perturbation theory (see [7], [13], and [17]). In recent years, the study of backward errors and residual bounds of matrix equations has been developed considerably. Taking full account of the special structure of the Sylvester equation, Higham [6] evaluates the backward error of an approximate solution to the matrix equation and determines the sensitivity of the equation to perturbations in the data. After that, Kågström [9] evaluates the normwise backward error of an approximate solution to the generalized Sylvester equation, and determines the sensitivity of the equation; Ghavimi and Laub [4] present a new backward error criterion, together with a sensitivity measure, for assessing solution accuracy of nonsymmetric and symmetric continuous-time algebraic Riccati equations. Normwise backward errors and residual bounds for continuous-time and discrete-time algebraic Riccati equations are obtained by the author [14], [15], [16]. This work, as a generalization of the results given by [15] and [16], derives normwise backward errors and residual bounds for an approximate Hermitian p.s.d. solution set to the P-DARE (1.1).

We begin in section 2 with pd-stable matrices and the Hermitian p.s.d. stabilizing solution set to the P-DARE (1.1). In sections 3 and 4 we derive normwise backward errors and residual bounds for an approximate Hermitian p.s.d. solution set to the PDARE (1.1), respectively. The results will be illustrated by simple numerical examples in section 5 .

\section{Preliminaries.}

2.1. Notation. Throughout this paper, $\mathcal{C}_{n}$ and $\mathcal{H}_{n}$ denote the set of $n \times n$ complex and $n \times n$ Hermitian matrices, respectively, and $\mathcal{C}_{n}^{p}$ and $\mathcal{H}_{n}^{p}$ denote the $p$ tuple product spaces $\mathcal{C}_{n} \times \cdots \times \mathcal{C}_{n}$ and $\mathcal{H}_{n} \times \cdots \times \mathcal{H}_{n}$, respectively. $\bar{A}$ denotes the conjugate of a matrix $A, A^{T}$ denotes the transpose of $A$, and $A^{H}=\bar{A}^{T}$. I stands for the identity matrix, $I_{n}$ is the identity matrix of order $n$, and 0 is the null matrix. The set of all eigenvalues of $A$ is denoted by $\lambda(A)$. The spectral radius $\rho(A)$ is defined by $\rho(A)=\max \left\{\left|\lambda_{j}\right|: \lambda_{j} \in \lambda(A)\right\}$. An $n \times n$ matrix $\Phi$ is said to be dstable if $\rho(\Phi)<1$. The symbol \|\|$_{F}$ is the Frobenius norm, and \|\|$_{2}$ is the spectral norm and the Euclidean vector norm. For $A=\left(a_{1}, \ldots, a_{n}\right)=\left(\alpha_{i j}\right) \in \mathcal{C}_{n}$ and a matrix $B, A \otimes B=\left(\alpha_{i j} B\right)$ is a Kronecker product, and $\operatorname{vec} A$ is a vector defined by $\operatorname{vec} A=\left(a_{1}^{T}, \ldots, a_{n}^{T}\right)^{T}$. For $A \in \mathcal{C}_{n}$ we have [5, pp. 32-34]

$$
\operatorname{vec} A^{T}=\Pi \operatorname{vec} A \text {, }
$$

where $\Pi$ is the vec-permutation matrix which can be expressed by

$$
\Pi=\sum_{k, l=1}^{n} e_{k} e_{l}^{T} \otimes e_{l} e_{k}^{T},
$$

in which $e_{k}$ denotes the $k$ th column of $I_{n}$. In order to save the space of the matrix representation, we use the following notation [12]:

$$
\operatorname{diag}\left\{N_{j}\right\}_{j=1}^{p}=\left(\begin{array}{ccc}
N_{1} & \cdots & 0 \\
\vdots & \ddots & \vdots \\
0 & \cdots & N_{p}
\end{array}\right), \quad \operatorname{cyc}\left\{N_{j}\right\}_{j=1}^{p}=\left(\begin{array}{cccc}
0 & \cdots & 0 & N_{1} \\
N_{2} & \ddots & & 0 \\
\vdots & \ddots & \ddots & \vdots \\
0 & \cdots & N_{p} & 0
\end{array}\right) .
$$


2.2. On pd-stable matrices. We first cite some definitions from [12].

Let $\Phi_{1}, \ldots, \Phi_{p} \in \mathcal{C}_{n}$. If there are complex numbers $\alpha_{1}, \ldots, \alpha_{p}$ such that

$$
\operatorname{det}\left[\operatorname{diag}\left\{\alpha_{j} I\right\}_{j=1}^{p}-\operatorname{cyc}\left\{\Phi_{j}\right\}_{j=1}^{p}\right]=0,
$$

then $\alpha_{1} \cdots \alpha_{p}$ is an eigenvalue of the matrix set $\left\{\Phi_{j}\right\}_{j=1}^{p}$.

The set of all eigenvalues of $\left\{\Phi_{j}\right\}_{j=1}^{p}$ is denoted by $\lambda\left(\left\{\Phi_{j}\right\}_{j=1}^{p}\right)$. We have [12]

$$
\lambda\left(\left\{\Phi_{j}\right\}_{j=1}^{p}\right)=\lambda\left(\Phi_{p} \Phi_{p-1} \cdots \Phi_{1}\right) .
$$

Consequently, if we define the spectral radius $\rho\left(\left\{\Phi_{j}\right\}_{j=1}^{p}\right)$ by

$$
\rho\left(\left\{\Phi_{j}\right\}_{j=1}^{p}\right)=\max \left\{\left|\lambda_{j}\right|: \lambda_{j} \in \lambda\left(\left\{\Phi_{j}\right\}_{j=1}^{p}\right)\right\},
$$

then

$$
\rho\left(\left\{\Phi_{j}\right\}_{j=1}^{p}\right)=\rho\left(\Phi_{p} \Phi_{p-1} \cdots \Phi_{1}\right) .
$$

Let $\Phi_{1}, \ldots, \Phi_{p} \in \mathcal{C}_{n}$. The matrix $p$-tuple $\left\{\Phi_{j}\right\}_{j=1}^{p}$ is said to be pd-stable if the matrix $\Phi_{p} \Phi_{p-1} \cdots \Phi_{1}$ is d-stable.

Let $\Phi_{1}, \ldots, \Phi_{p} \in \mathcal{C}_{n}$. Define the linear operator $\mathbf{L}: \mathcal{H}_{n}^{p} \rightarrow \mathcal{H}_{n}^{p}$ by

$$
\begin{gathered}
\mathbf{L}\left(W_{1}, \ldots, W_{p}\right)=\left(W_{1}-\Phi_{2}^{H} W_{2} \Phi_{2}, \ldots, W_{p-1}-\Phi_{p}^{H} W_{p} \Phi_{p}, W_{p}-\Phi_{1}^{H} W_{1} \Phi_{1}\right), \\
\left(W_{1}, \ldots, W_{p}\right) \in \mathcal{H}_{n}^{p} .
\end{gathered}
$$

It is known [12] that the matrix $L$ defined by

$$
L=I_{p n^{2}}-\left(\begin{array}{cccc}
0 & \Phi_{2}^{T} \otimes \Phi_{2}^{H} & \ldots & 0 \\
\vdots & \ddots & \ddots & \vdots \\
\vdots & & \ddots & \Phi_{p}^{T} \otimes \Phi_{p}^{H} \\
\Phi_{1}^{T} \otimes \Phi_{1}^{H} & \ldots & \ldots & 0
\end{array}\right)
$$

is a matrix representation of $\mathbf{L}$ on the space

$$
\mathcal{H}^{p n^{2}} \equiv\left\{\left(w_{1}^{T}, \ldots, w_{p}^{T}\right)^{T}: w_{j}=\operatorname{vec} W_{j}, W_{j} \in \mathcal{H}_{n} \quad \forall j\right\} .
$$

Lemma 2.1 (see [12, Lemma 2.1]). The linear operator $\mathbf{L}$ defined by (2.1) is singular provided that there is an eigenvalue $\lambda_{k} \in \lambda\left(\left\{\Phi_{j}\right\}_{j=1}^{p}\right)$ with $\left|\lambda_{k}\right|=1$.

Lemma 2.2 (see [12, Lemma 2.2]). Let $\Phi=\operatorname{cyc}\left\{\Phi_{j}\right\}_{j=1}^{p}$, where $\Phi_{j} \in \mathcal{C}_{n} \forall j$. If $\left\{\Phi_{j}\right\}_{j=1}^{p}$ is pd-stable, then $\Phi$ is d-stable.

Assume that $\left\{\Phi_{j}\right\}_{j=1}^{p}$ is pd-stable. By Lemma 2.2 the matrix $L$ defined by (2.2) is nonsingular, and thus $\mathbf{L}^{-1}$ exists. In such a case, we define the quantity $l$ by

$$
l=\left\|\mathbf{L}^{-1}\right\|^{-1},
$$

where the operator norm \|\| for $\mathbf{L}^{-1}$ is induced by the Frobenius norm \|\|$_{F}$ on $\mathcal{H}_{n}^{p}$. Note that $\mathcal{H}_{n}^{p}$ is not a subspace of $\mathcal{C}_{n}^{p}$, but by [12, Appendix (I)] we have

$$
l=\left\|L^{-1}\right\|_{2}^{-1},
$$


i.e., the induced operator norms of $\mathbf{L}^{-1}$ on $\mathcal{C}_{n}^{p}$ and $\mathcal{H}_{n}^{p}$ are equal.

Let the matrix set $\left\{\Phi_{j}\right\}_{j=1}^{p}$ be pd-stable. Define

$$
s_{\mathrm{pd}}=\min \left\{\max _{1 \leq j \leq p}\left\|E_{j}\right\|_{2}: \rho\left(\left\{\left(I-E_{j}\right)^{-1} \Phi_{j}\right\}_{j=1}^{p}\right)=1, E_{j} \in \mathcal{C}_{n} \quad \forall j\right\} .
$$

The quantity $s_{\text {pd }}$ measures the smallest $\max _{1 \leq j \leq p}\left\|E_{j}\right\|_{2}$ such that $\left\{\left(I-E_{j}\right)^{-1} \Phi_{j}\right\}_{j=1}^{p}$ has an eigenvalue on the unit circle. Note that the computation of $s_{\mathrm{pd}}$ may be a rather difficult computational problem in the general case.

Lemma 2.3. Let $\left\{\Phi_{j}\right\}_{j=1}^{p}$ be pd-stable, and let $\mathbf{L}$ be the linear operator defined by (2.1) with $L$ of (2.2) as its matrix representation. Let $l$ and $s_{\mathrm{pd}}$ be the quantities defined by (2.3) and (2.4), respectively, and let

$$
\phi_{j}=\left\|\Phi_{j}\right\|_{2}, \quad \phi=\max _{1 \leq j \leq p} \phi_{j} .
$$

Then

$$
\frac{l}{\phi^{2}+\phi \sqrt{\phi^{2}+l}+l} \leq s_{\mathrm{pd}}
$$

Proof. Let the matrices $E_{j}^{*} \in \mathcal{C}_{n}(j=1, \ldots, p)$ satisfy

$$
s_{\mathrm{pd}}=\max _{1 \leq j \leq p}\left\|E_{j}^{*}\right\|_{2} \quad \text { with } \quad \rho\left(\left\{\left(I-E_{j}^{*}\right)^{-1} \Phi_{j}\right\}_{j=1}^{p}\right)=1 .
$$

By Lemma 2.1 the transformation

$$
\left(\begin{array}{c}
W_{1} \\
\vdots \\
W_{p-1} \\
W_{p}
\end{array}\right) \mapsto\left(\begin{array}{c}
W_{1}-\left[\left(I-E_{2}^{*}\right)^{-1} \Phi_{2}\right]^{H} W_{2}\left[\left(I-E_{2}^{*}\right)^{-1} \Phi_{2}\right] \\
\vdots \\
W_{p-1}-\left[\left(I-E_{p}^{*}\right)^{-1} \Phi_{p}\right]^{H} W_{p}\left[\left(I-E_{p}^{*}\right)^{-1} \Phi_{p}\right] \\
W_{p}-\left[\left(I-E_{1}^{*}\right)^{-1} \Phi_{1}\right]^{H} W_{1}\left[\left(I-E_{1}^{*}\right)^{-1} \Phi_{1}\right]
\end{array}\right)
$$

is singular, where $W_{j} \in \mathcal{H}_{n}$ for all $j$; i.e., there are Hermitian matrices $W_{1}^{*}, \ldots, W_{p}^{*}$ with $W_{k} \neq 0$ for some index $k \in\{1, \ldots, p\}$ such that

$$
\begin{gathered}
W_{1}^{*}-\left[\left(I-E_{2}^{*}\right)^{-1} \Phi_{2}\right]^{H} W_{2}^{*}\left[\left(I-E_{2}^{*}\right)^{-1} \Phi_{2}\right]=0, \\
\vdots \\
W_{p}^{*}-\left[\left(I-E_{1}^{*}\right)^{-1} \Phi_{1}\right]^{H} W_{1}^{*}\left[\left(I-E_{1}^{*}\right)^{-1} \Phi_{1}\right]=0 .
\end{gathered}
$$

Let $N_{j} \in \mathcal{C}_{n}$ be defined by

$$
I+N_{j}=\left(I-E_{j}^{*}\right)^{-1}, \quad j=1, \ldots, p .
$$

Then (2.7) can be written as

$$
\mathbf{L}\left(\begin{array}{c}
W_{1}^{* T} \\
\vdots \\
W_{p}^{* T}
\end{array}\right)^{T}=\left(\begin{array}{c}
\left(\Phi_{2}^{H} W_{2}^{*} N_{2} \Phi_{2}+\Phi_{2}^{H} N_{2}^{H} W_{2}^{*} \Phi_{2}+\Phi_{2}^{H} N_{2}^{H} W_{2}^{*} N_{2} \Phi_{2}\right)^{T} \\
\vdots \\
\left(\Phi_{1}^{H} W_{1}^{*} N_{1} \Phi_{1}+\Phi_{1}^{H} N_{1}^{H} W_{1}^{*} \Phi_{1}+\Phi_{1}^{H} N_{1}^{H} W_{1}^{*} N_{1} \Phi_{1}\right)^{T}
\end{array}\right)^{T},
$$


or equivalently, by letting $\operatorname{vec} W_{j}^{*}=w_{j}^{*}(j=1, \ldots, p)$, we have

$$
L\left(\begin{array}{c}
w_{1}^{*} \\
\vdots \\
w_{p}^{*}
\end{array}\right)=\left(\operatorname{cyc}\left\{\Omega_{j}^{T}\right\}_{j=1}^{p}\right)^{T}\left(\begin{array}{c}
w_{1}^{*} \\
\vdots \\
w_{p}^{*}
\end{array}\right),
$$

where

$$
\Omega_{j}=\left(\Phi_{j}^{T} N_{j}^{T}\right) \otimes \Phi_{j}^{H}+\Phi_{j}^{T} \otimes\left(\Phi_{j}^{H} N_{j}^{H}\right)+\left(\Phi_{j}^{T} N_{j}^{T}\right) \otimes\left(\Phi_{j}^{H} N_{j}^{H}\right) \quad \forall j .
$$

Inverting $L$ and taking 2-norm of the two sides of (2.10) we get

$$
\nu^{2}+2 \nu-\frac{l}{\phi^{2}} \geq 0
$$

where

$$
\nu=\max _{1 \leq j \leq p} \nu_{j} \quad \text { with } \quad \nu_{j}=\left\|N_{j}\right\|_{2} \forall j
$$

and by (2.11),

$$
\nu \geq \sqrt{1+\frac{l}{\phi^{2}}}-1=\frac{l}{\phi^{2}+\phi \sqrt{\phi^{2}+l}} .
$$

Observe that the relations of (2.8) imply

$$
N_{j}=\left(I+N_{j}\right) E_{j}^{*}
$$

and

$$
\left\|N_{j}\right\|_{2} \leq\left(1+\left\|N_{j}\right\|_{2}\right)\left\|E_{j}^{*}\right\|_{2} .
$$

Hence, we have

$$
\left\|E_{j}^{*}\right\|_{2} \geq \frac{\left\|N_{j}\right\|_{2}}{1+\left\|N_{j}\right\|_{2}}
$$

and

$$
\max _{1 \leq j \leq p}\left\|E_{j}^{*}\right\|_{2} \geq \frac{\max _{1 \leq j \leq p}\left\|N_{j}\right\|_{2}}{1+\max _{1 \leq j \leq p}\left\|N_{j}\right\|_{2}}=\frac{\nu}{1+\nu} .
$$

Combining it with (2.13) gives the inequality (2.6).

From Lemma 2.3 we get the following lemma.

Lemma 2.4. Let $\left\{\Phi_{j}\right\}_{j=1}^{p}$ be pd-stable, and let $\mathbf{L}$ be the linear operator defined by (2.1) with $L$ in (2.2) as its matrix representation. Moreover, let $l$ and $\phi$ be defined by (2.3) and (2.5), respectively. If $E_{j} \in \mathcal{C}_{n}(j=1, \ldots, p)$ satisfy

$$
\max _{1 \leq j \leq p}\left\|E_{j}\right\|_{2}<\frac{l}{\phi^{2}+\phi \sqrt{\phi^{2}+l}+l},
$$

then the matrix set $\left\{\left(\Phi_{j}+E_{j}\right)\right\}_{j=1}^{p}$ is pd-stable. 
2.3. The Hermitian p.s.d. stabilizing solution set. By [2], the matrix pair sets $\left\{\left(A_{j}, B_{j}\right)\right\}_{j=1}^{p}$ and $\left\{\left(A_{j}, C_{j}\right)\right\}_{j=1}^{p}$ are said to be pd-stabilizable and pd-detectable, respectively, if the pairs $\left(\mathcal{A}_{j}, \mathcal{B}_{j}\right)$ and $\left(\mathcal{A}_{j}, \mathcal{C}_{j}\right)$ are d-stabilizable and d-detectable, respectively, for $j=1, \ldots, p$, where

$$
\begin{aligned}
& \mathcal{A}_{j}=A_{\pi_{j}(p)} \cdots A_{\pi_{j}(1)}, \\
& \mathcal{B}_{j}=\left(A_{\pi_{j}(p)} \cdots A_{\pi_{j}(2)} B_{\pi_{j}(1)}, A_{\pi_{j}(p)} \cdots A_{\pi_{j}(3)} B_{\pi_{j}(2)}, \ldots, A_{\pi_{j}(p)} B_{\pi_{j}(p-1)}, B_{\pi_{j}(p)}\right), \\
& \mathcal{C}_{j}=\left(C_{\pi_{j}(1)}^{T}, A_{\pi_{j}(1)}^{T} C_{\pi_{j}(2)}^{T}, A_{\pi_{j}(1)}^{T} A_{\pi_{j}(2)}^{T} C_{\pi_{j}(3)}^{T}, \ldots, A_{\pi_{j}(1)}^{T} \cdots A_{\pi_{j}(p-1)}^{T} C_{\pi_{j}(p)}^{T}\right)^{T}
\end{aligned}
$$

and $\pi_{j}($ ) is a permutation defined by

$$
\pi_{j}(k)= \begin{cases}k-j+1+p & \text { for } k=1, \ldots, j-1 \text { and } j \geq 2 \\ k-j+1 & \text { for } k=j, \ldots, p\end{cases}
$$

Note that the pair $(A, B)$ is d-stabilizable if $w^{H} B=0$ and $w^{H} A=\lambda w^{H}$ for some constant $\lambda$ implies $|\lambda|<1$ or $w=0$, and that the pair $(A, C)$ is d-detectable if $\left(A^{H}, C^{H}\right)$ is d-stabilizable.

Let $X_{j} \in \mathcal{H}_{n}(j=1, \ldots, p)$ and $\left\{X_{j}\right\}_{j=1}^{p}$ be a solution set to the P-DARE (1.1). If the matrix set $\left\{\left(I+G_{j} X_{j}\right)^{-1} A_{j}\right\}_{j=1}^{p}$ is pd-stable, then $\left\{X_{j}\right\}_{j=1}^{p}$ is said to be a stabilizing solution set to (1.1). If $X_{j} \geq 0$ for all $j$, then $\left\{X_{j}\right\}_{j=1}^{p}$ is said to be a Hermitian p.s.d. solution set.

The following result is a basic result on the existence and uniqueness of Hermitian p.s.d. stabilizing solution sets to the P-DARE (1.1). (See [1], [2], [12].)

Theorem 2.5. For the P-DARE (1.1), if $\left\{\left(A_{j}, B_{j}\right)\right\}_{j=1}^{p}$ and $\left\{\left(A_{j}, C_{j}\right)\right\}_{j=1}^{p}$ are pd-stabilizable and pd-detectable, respectively, then there is a unique Hermitian p.s.d. stabilizing solution set $\left\{X_{j}\right\}_{j=1}^{p}$ to the P-DARE (1.1).

The result will be illustrated by Example 5.1 of section 5 .

Throughout this paper, the matrix pair sets $\left\{\left(A_{j}, B_{j}\right)\right\}_{j=1}^{p}$ and $\left\{\left(A_{j}, C_{j}\right)\right\}_{j=1}^{p}$ of (1.1) are assumed to be pd-stabilizable and pd-detectable, respectively.

\section{Backward errors.}

3.1. Definitions. Let $\left\{\tilde{X}_{j}\right\}_{j=1}^{p}$ approximate the unique Hermitian p.s.d. stabilizing solution set to the P-DARE (1.1), and assume that the matrices $I+G_{j} \tilde{X}_{j}(j=$ $1, \ldots, p)$ are nonsingular. Moreover, let $\Delta A_{j}, \Delta G_{j}, \Delta H_{j}$ be the corresponding perturbations in the coefficient matrices $A_{j}, G_{j}, H_{j}(j=1, \ldots, p)$ of (1.1), respectively. The normwise backward error $\eta\left(\left\{\tilde{X}_{j}\right\}_{j=1}^{p}\right)$ of the approximate solution set $\left\{\tilde{X}_{j}\right\}_{j=1}^{p}$ can be defined by

$$
\eta\left(\left\{\tilde{X}_{j}\right\}_{j=1}^{p}\right)=\max _{1 \leq j \leq p} \min _{\left\{\left(\Delta A_{j}, \Delta G_{j}, \Delta H_{j}\right)\right\}_{j=1}^{p} \in \mathcal{E}}\left\|\left(\frac{\Delta A_{j}}{\alpha_{j}}, \frac{\Delta G_{j}}{\beta_{j}}, \frac{\Delta H_{j}}{\gamma_{j}}\right)\right\|_{F},
$$


where the set $\mathcal{E}$ is defined by

$$
\mathcal{E}=\left\{\left\{\left(\Delta A_{j}, \Delta G_{j}, \Delta H_{j}\right)\right\}_{j=1}^{p}:=\left(A_{j}+\Delta A_{j}\right)^{H} \tilde{X}_{j}\left[I+\left(G_{j}+\Delta G_{j}\right) \tilde{X}_{j}\right]^{-1}\left(A_{j}+\Delta A_{j}\right)\right\},
$$

and $\alpha_{j}, \beta_{j}, \gamma_{j}(j=1, \ldots, p)$ are positive parameters. Taking $\alpha_{j}=\beta_{j}=\gamma_{j}=1$ for $j=1, \ldots, p$ yields the normwise absolute backward error $\eta_{\text {abs }}\left(\left\{\tilde{X}_{j}\right\}_{j=1}^{p}\right)$, and taking $\alpha_{j}=\left\|A_{j}\right\|_{F}, \beta_{j}=\left\|G_{j}\right\|_{F}, \gamma_{j}=\left\|H_{j}\right\|_{F}(j=1, \ldots, p)$ yields the normwise relative backward error $\eta_{\text {rel }}\left(\left\{\tilde{X}_{j}\right\}_{j=1}^{p}\right)$.

From (3.1) and (3.2) we see that the backward error $\eta\left(\left\{\tilde{X}_{j}\right\}_{j=1}^{p}\right)$ of an approximate Hermitian solution set $\left\{\tilde{X}_{j}\right\}_{j=1}^{p}$ to the P-DARE (1.1) is a measure of "smallest" perturbations $\Delta A_{j} / \alpha_{j}, \Delta G_{j} / \beta_{j}, \Delta H_{j} / \gamma_{j}(j=1, \ldots, p)$ such that $\left\{\tilde{X}_{j}\right\}_{j=1}^{p}$ is just a Hermitian solution set to the perturbed P-DARE

$$
\begin{gathered}
\tilde{X}_{j-1}=\left(A_{j}+\Delta A_{j}\right)^{H} \tilde{X}_{j}\left[I+\left(G_{j}+\Delta G_{j}\right) \tilde{X}_{j}\right]^{-1}\left(A_{j}+\Delta A_{j}\right)+H_{j}+\Delta H_{j}, \\
j=1, \ldots, p .
\end{gathered}
$$

Moreover, from (3.1) and (3.2) we see that

$$
\eta\left(\left\{\tilde{X}_{j}\right\}_{j=1}^{p}\right)=\max _{1 \leq j \leq p} \eta_{j}
$$

where each $\eta_{j}$ is defined by

$$
\eta_{j}=\min _{\left(\Delta A_{j}, \Delta G_{j}, \Delta H_{j}\right) \in \mathcal{E}_{j}}\left\|\left(\frac{\Delta A_{j}}{\alpha_{j}}, \frac{\Delta G_{j}}{\beta_{j}}, \frac{\Delta H_{j}}{\gamma_{j}}\right)\right\|_{F}
$$

in which the set $\mathcal{E}_{j}$ is defined by

$$
\mathcal{E}_{j}=\left\{\left(\Delta A_{j}, \Delta G_{j}, \Delta H_{j}\right): \begin{array}{l}
\Delta A_{j} \in \mathcal{C}_{n}, \Delta G_{j}, \Delta H_{j} \in \mathcal{H}_{n}, \\
= \\
\\
\quad \tilde{X}_{j-1} \\
\\
\\
\left.+H_{j}+\Delta A_{j}\right)^{H} \tilde{X}_{j}\left[I+\left(G_{j}+\Delta G_{j}\right) \tilde{X}_{j}\right]^{-1}\left(A_{j}+\Delta A_{j}\right)
\end{array}\right\} .
$$

Consequently, the problem of estimating the backward error $\eta\left(\left\{\tilde{X}_{j}\right\}_{j=1}^{p}\right)$ is reduced to the problem of estimating $\eta_{j}$ for $j=1, \ldots, p$. 
3.2. Estimates of $\boldsymbol{\eta}_{\boldsymbol{j}}(\boldsymbol{j}=\mathbf{1}, \ldots, \boldsymbol{p})$. For each $j \in\{1, \ldots, p\}$ define

$$
\tilde{L}_{j}=\tilde{X}_{j}\left(I+G_{j} \tilde{X}_{j}\right)^{-1} \in \mathcal{H}_{n}, \quad \tilde{K}_{j}=\tilde{L}_{j} A_{j} \in \mathcal{C}_{n},
$$

and define the residual $\widehat{R}_{j}$ by

$$
\widehat{R}_{j}=\tilde{X}_{j-1}-A_{j}^{H} \tilde{X}_{j}\left(I+G_{j} \tilde{X}_{j}\right)^{-1} A_{j}-H_{j},
$$

where $\tilde{X}_{0}=\tilde{X}_{p}$. Moreover, define

$$
\begin{aligned}
& q_{j}\left(\Delta A_{j}, \Delta G_{j}\right) \\
& =-\tilde{K}_{j}^{H} \Delta G_{j} \tilde{L}_{j} \Delta G_{j}\left(I+\tilde{L}_{j} \Delta G_{j}\right)^{-1} \tilde{K}_{j}+\tilde{K}_{j}^{H} \Delta G_{j}\left(I+\tilde{L}_{j} \Delta G_{j}\right)^{-1} \tilde{L}_{j} \Delta A_{j} \\
& \quad+\Delta A_{j}^{H} \tilde{L}_{j} \Delta G_{j}\left(I+\tilde{L}_{j} \Delta G_{j}\right)^{-1} \tilde{K}_{j}-\Delta A_{j}^{H}\left(I+\tilde{L}_{j} \Delta G_{j}\right)^{-1} \tilde{L}_{j} \Delta A_{j} .
\end{aligned}
$$

Then by $[15$, section 2$]$, the $j$ th equation of $(3.3)$ is equivalent to

$$
\tilde{K}_{j}^{H} \Delta A_{j}+\Delta A_{j}^{H} \tilde{K}_{j}-\tilde{K}_{j}^{H} \Delta G_{j} \tilde{K}_{j}+\Delta H_{j}=\widehat{R}_{j}+q_{j}\left(\Delta A_{j}, \Delta G_{j}\right) .
$$

3.2.1. The real case. We now consider the case that all the coefficient matrices $A_{j}, G_{j}, H_{j}$; the perturbations $\Delta A_{j}, \Delta G_{j}, \Delta H_{j}$; and the approximate solution set $\left\{\tilde{X}_{j}\right\}_{j=1}^{p}$ are real. In such a case, $(3.10)$ can be written as

$$
\tilde{K}_{j}^{T} \Delta A_{j}+\Delta A_{j}^{T} \tilde{K}_{j}-\tilde{K}_{j}^{T} \Delta G_{j} \tilde{K}_{j}+\Delta H_{j}=\widehat{R}_{j}+q_{j}\left(\Delta A_{j}, \Delta G_{j}\right)
$$

Define the matrix $T_{j}$ by

$$
T_{j}=\left(\alpha_{j}\left[I_{n} \otimes \tilde{K}_{j}^{T}+\left(\tilde{K}_{j}^{T} \otimes I_{n}\right) \Pi\right],-\beta_{j} \tilde{K}_{j}^{T} \otimes \tilde{K}_{j}^{T}, \gamma_{j} I_{n^{2}}\right)
$$

where $\Pi$ is the vec-permutation matrix. Then (3.11) is equivalent to the nonlinear system

$$
T_{j}\left(\begin{array}{c}
\frac{\operatorname{vec} \Delta A_{j}}{\alpha_{j}} \\
\frac{\operatorname{vec} \Delta G_{j}}{\beta_{j}} \\
\frac{\operatorname{vec} \Delta H_{j}}{\gamma_{j}}
\end{array}\right)=\operatorname{vec} \widehat{R}_{j}+\operatorname{vec} q_{j}\left(\Delta A_{j}, \Delta G_{j}\right) .
$$

By using the technique described by [15, section 2] we can prove the following result.

TheOREm 3.1. For each $j \in\{1, \ldots, p\}$, let $T_{j}$ be the matrix defined by (3.12), and define $\tau_{j}, \rho_{j}, \mu_{j}$, and $\nu_{j}$ by

$$
\begin{aligned}
& \tau_{j}=\left\|T_{j}^{\dagger}\right\|_{2}, \quad \rho_{j}=\left\|T_{j}^{\dagger} \operatorname{vec} \widehat{R}_{j}\right\|_{2}, \\
& \mu_{j}=\left(\alpha_{j}^{2}+\beta_{j}^{2}\left\|\tilde{K}_{j}\right\|_{2}^{2}\right)\|\tilde{L}\|_{2}, \quad \nu_{j}=\beta_{j}\left\|\tilde{X}_{j}\right\|_{2}\left\|\left(I+G_{j} \tilde{X}_{j}\right)^{-1}\right\|_{2},
\end{aligned}
$$


where $T_{j}^{\dagger}$ denotes the Moore-Penrose inverse of $T_{j}$, and $\tilde{L}_{j}, \tilde{K}_{j}$, and $\widehat{R}_{j}$ are the $m a$ trices defined by (3.7) and (3.8). If

$$
\rho_{j} \leq \min \left\{\frac{1}{\nu_{j}}, \frac{\tau_{j}}{\tau_{j} \nu_{j}+2 \mu_{j}+\sqrt{\left(\tau_{j} \nu_{j}+2 \mu_{j}\right)^{2}-\tau_{j}^{2} \nu_{j}^{2}}}\right\},
$$

then

$$
l_{j} \leq \eta_{j} \leq u_{j}
$$

where

$$
\begin{aligned}
& u_{j}=\frac{2 \tau_{j} \rho_{j}}{\tau_{j}\left(1+\nu_{j} \rho_{j}\right)+\sqrt{\tau_{j}^{2}\left(1+\nu_{j} \rho_{j}\right)^{2}-4 \tau_{j}\left(\tau_{j} \nu_{j}+\mu_{j}\right) \rho_{j}}}, \\
& l_{j}=\rho_{j}-\frac{\mu_{j} u_{j}^{2}}{\tau_{j}\left(1-\nu_{j} u_{j}\right)} .
\end{aligned}
$$

From Theorem 3.1 and the relation (3.4) we get the nonlinear estimates

$$
l^{*} \equiv \max _{1 \leq j \leq p} l_{j} \leq \eta\left(\left\{\tilde{X}_{j}\right\}_{j=1}^{p}\right) \leq \max _{1 \leq j \leq p} u_{j} \equiv u^{*}
$$

Note that

$$
u_{j}=\rho_{j}+\frac{\mu_{j}}{\tau_{j}} \rho_{j}^{2}+O\left(\rho^{3}\right), \quad l_{j}=\rho_{j}-\frac{\mu_{j}}{\tau_{j}} \rho_{j}^{2}+O\left(\rho^{3}\right), \quad j=1, \ldots, p .
$$

Consequently, we have the linear estimates

$$
\eta_{j} \approx \rho_{j} \forall j, \quad \text { and } \quad \eta\left(\left\{\tilde{X}_{j}\right\}_{j=1}^{p}\right) \approx \max _{1 \leq j \leq p} \rho_{j}
$$

as $\max _{1 \leq j \leq p} \rho_{j} \rightarrow 0(j \rightarrow \infty)$.

3.2.2. The complex case. Let

$$
\begin{aligned}
& \alpha_{j}\left[I_{n} \otimes \tilde{K}_{j}^{H}+\left(\tilde{K}_{j}^{T} \otimes I_{n}\right) \Pi\right]=U_{j, 1}+i \Omega_{j, 1}, \\
& -\beta_{j} \tilde{K}^{T} \otimes \tilde{K}_{j}^{H}=U_{j, 2}+i \Omega_{j, 2}, \\
& \operatorname{vec} \Delta A_{j}=x_{j}+i y_{j}, \quad \operatorname{vec} \Delta G_{j}=u_{j}+i v_{j}, \quad \operatorname{vec} \Delta H_{j}=z_{j}+i w_{j}, \\
& \operatorname{vec} \widehat{R}_{j}=r_{j}+i s_{j}, \quad \operatorname{vec} q_{j}\left(\Delta A_{j}, \Delta G_{j}\right)=a_{j}+i b_{j}, \quad i=\sqrt{-1},
\end{aligned}
$$

where $U_{j, k}$ and $\Omega_{j, k}(k=1,2)$ are real matrices, and $x_{j}, y_{j}, u_{j}, v_{j}, z_{j}, w_{j}, r_{j}, s_{j}, a_{j}, b_{j}$ are real vectors. Moreover, let

$$
T_{j}^{(c)}=\left(\begin{array}{cccccc}
U_{j, 1} & -\Omega_{j, 1} & U_{j, 2} & -\Omega_{j, 2} & \gamma_{j} I_{n^{2}} & 0 \\
\Omega_{j, 1} & U_{j, 1} & \Omega_{j, 2} & U_{j, 2} & 0 & \gamma_{j} I_{n^{2}}
\end{array}\right),
$$


and

$$
\chi_{j}=\left(\frac{x_{j}^{T}}{\alpha_{j}}, \frac{y_{j}^{T}}{\alpha_{j}}, \frac{u_{j}^{T}}{\beta_{j}}, \frac{v_{j}^{T}}{\beta_{j}}, \frac{z_{j}^{T}}{\gamma_{j}}, \frac{w_{j}^{T}}{\gamma_{j}}\right)^{T} .
$$

Then (3.10) is equivalent to

$$
T_{j}^{(c)} \chi_{j}=\left(\begin{array}{c}
r_{j} \\
s_{j}
\end{array}\right)+\left(\begin{array}{c}
a_{j} \\
b_{j}
\end{array}\right) .
$$

Referring to [10], [11], and the proof of Theorem 3.1, we can prove the following result.

Theorem 3.2. For each $j \in\{1, \ldots, p\}$, let $T_{j}^{(c)}$ be the matrix defined by (3.20). Define $\mu_{j}$ and $\nu_{j}$ by (3.14), and define $\tau_{j}^{(c)}$ and $\rho_{j}^{(c)}$ by

$$
\tau_{j}^{(c)}=\left\|T_{j}^{(c)^{\dagger}}\right\|_{2}, \quad \rho_{j}^{(c)}=\left\|T_{j}^{(c)^{\dagger}}\left(\begin{array}{c}
r_{j} \\
s_{j}
\end{array}\right)\right\|_{2} .
$$

If

$$
\rho_{j}^{(c)} \leq \min \left\{\frac{1}{\nu_{j}}, \frac{\tau_{j}^{(c)}}{\tau_{j}^{(c)} \nu_{j}+2 \mu_{j}+\sqrt{\left(\tau_{j}^{(c)} \nu_{j}+2 \mu_{j}\right)^{2}-\tau_{j}^{(c)} \nu^{2}}}\right\},
$$

then we have

$$
l_{j}^{(c)} \leq \eta_{j} \leq u_{j}^{(c)}
$$

where

$$
\begin{aligned}
& u_{j}^{(c)}=\frac{2 \tau_{j}^{(c)} \rho_{j}^{(c)}}{\tau_{j}^{(c)}\left(1+\nu_{j} \rho_{j}^{(c)}\right)+\sqrt{\tau_{j}^{(c)^{2}}\left(1+\nu_{j} \rho_{j}^{(c)}\right)^{2}-4 \tau_{j}^{(c)}\left(\tau_{j}^{(c)} \nu_{j}+\mu_{j}\right) \rho_{j}^{(c)}}}, \\
& l_{j}^{(c)}=\rho_{j}^{(c)}-\frac{\mu_{j} u_{j}^{(c)}{ }^{2}}{\tau_{j}^{(c)}\left(1-\nu_{j} u_{j}^{(c)}\right)} .
\end{aligned}
$$

From Theorem 3.2 and the relation (3.4) we get

$$
l^{(c)} \equiv \max _{1 \leq j \leq p} l_{j}^{(c)} \leq \eta\left(\left\{\tilde{X}_{j}\right\}_{j=1}^{p}\right) \leq \max _{1 \leq j \leq p} u_{j}^{(c)} \equiv u^{(c)} .
$$

4. Residual bounds. In this section we prove the following result.

THEOREM 4.1. Let $\left\{\tilde{X}_{j}\right\}_{j=1}^{p}$ be an approximate Hermitian solution set to the $P$-DARE (1.1) such that the matrices $I+G_{j} \tilde{X}_{j}(j=1, \ldots, p)$ are nonsingular, and the matrix set $\left\{\left(I+G_{j} \tilde{X}_{j}\right)^{-1} A_{j}\right\}_{j=1}^{p}$ is pd-stable. Define the residuals $\widehat{R}_{j}$ by

$$
\widehat{R}_{j}=\tilde{X}_{j-1}-A_{j}^{H} \tilde{X}_{j}\left(I+G_{j} \tilde{X}_{j}\right)^{-1} A_{j}-H_{j}, \quad j=1, \ldots, p,
$$

where $\tilde{X}_{0}=\tilde{X}_{p}$, and define the linear operator $\mathbf{L}: \mathcal{H}_{n}^{p} \rightarrow \mathcal{H}_{n}^{p}$ by

$$
\mathbf{L}\left(W_{1}, \ldots, W_{p-1}, W_{p}\right)=\left(W_{1}-\tilde{\Phi}_{2}^{H} W_{2} \tilde{\Phi}_{2}, \ldots, W_{p-1}-\tilde{\Phi}_{p}^{H} W_{p} \tilde{\Phi}_{p}, W_{p}-\tilde{\Phi}_{1}^{H} W_{1} \tilde{\Phi}_{1}\right),
$$


where $W_{1}, \ldots, W_{p} \in \mathcal{H}_{n}$, and the matrices $\tilde{\Phi}_{j}$ are defined by

$$
\tilde{\Phi}_{j}=\left(I+G_{j} \tilde{X}_{j}\right)^{-1} A_{j}, \quad j=1, \ldots, p .
$$

Moreover, let

$$
\begin{aligned}
& \phi=\max _{1 \leq j \leq p} \phi_{j} \quad \text { with } \quad \phi_{j}=\left\|\tilde{\Phi}_{j}\right\|_{2} \forall j, \\
& \gamma=\max _{1 \leq j \leq p} \gamma_{j} \quad \text { with } \quad \gamma_{j}=\left\|\left(I+G_{j} \tilde{X}_{j}\right)^{-1} G_{j}\right\|_{2} \quad \forall j,
\end{aligned}
$$

and

$$
l=\left\|\mathbf{L}^{-1}\right\|^{-1}, \quad \epsilon=\left\|\mathbf{L}^{-1}\left(\widehat{R}_{1}, \ldots, \widehat{R}_{p}\right)\right\|_{F},
$$

where the operator norm \|\| for $\mathbf{L}^{-1}$ is induced by the Frobenius norm \|\|$_{F}$ on $\mathcal{C}_{n}^{p}$. If

$$
\epsilon<\frac{l}{\gamma\left(2 \phi^{2}+2 \phi \sqrt{\phi^{2}+l}+l\right)},
$$

then for the unique Hermitian p.s.d. stabilizing solution set $\left\{X_{j}\right\}_{j=1}^{p}$ to the P-DARE (1.1) we have

$$
\left\|\left(\tilde{X}_{1}-X_{1}, \ldots, \tilde{X}_{p}-X_{p}\right)\right\|_{F} \leq \frac{2 l \epsilon}{(1+\gamma \epsilon) l+\sqrt{(1+\gamma \epsilon)^{2} l^{2}-4\left(\phi^{2}+l\right) \gamma l \epsilon}} \equiv r(\epsilon) .
$$

As a corollary of Theorem 4.1, we have the estimate

$$
\left\|\left(\tilde{X}_{1}-X_{1}, \ldots, \tilde{X}_{p}-X_{p}\right)\right\|_{F} \leq \frac{2 \epsilon}{1+\gamma \epsilon}=\frac{2\left\|\mathbf{L}^{-1}\left(\widehat{R}_{1}, \ldots, \widehat{R}_{p}\right)\right\|_{F}}{1+\gamma\left\|\mathbf{L}^{-1}\left(\widehat{R}_{1}, \ldots, \widehat{R}_{p}\right)\right\|_{F}} .
$$

Moreover, from (4.7) we obtain a relative error bound $b_{\text {rel }}\left(\tilde{X}_{j}\right)$ for each $\tilde{X}_{j}(1 \leq$ $j \leq p)$ :

$$
\frac{\left\|\tilde{X}_{j}-X_{j}\right\|_{F}}{\left\|X_{j}\right\|_{F}} \leq \frac{\|\tilde{X}-X\|_{F} /\left\|\tilde{X}_{j}\right\|_{F}}{1-\|\tilde{X}-X\|_{F} /\left\|\tilde{X}_{j}\right\|_{F}} \leq \frac{r(\epsilon) /\left\|\tilde{X}_{j}\right\|_{F}}{1-r(\epsilon) /\left\|\tilde{X}_{j}\right\|_{F}} \equiv b_{\text {rel }}\left(\tilde{X}_{j}\right) .
$$

Proof of Theorem 4.1. The proof is completed by the following three steps.

Step 1. Perturbation equation.

Let

$$
\begin{aligned}
& X=\operatorname{diag}\left\{X_{j}\right\}_{j=1}^{p}, \quad \tilde{X}=\operatorname{diag}\left\{\tilde{X}_{j}\right\}_{j=1}^{p}, \\
& \Delta X=\operatorname{diag}\left\{\Delta X_{j}\right\}_{j=1}^{p} \quad \text { with } \quad \Delta X_{j}=\tilde{X}_{j}-X_{j}, \quad j=1, \ldots, p, \\
& A=\operatorname{cyc}\left\{A_{j}\right\}_{j=1}^{p}, \quad G=\operatorname{diag}\left\{G_{j}\right\}_{j=1}^{p}, \\
& H=\operatorname{diag}\left(H_{2}, \ldots, H_{p}, H_{1}\right), \quad \widehat{R}=\operatorname{diag}\left(\widehat{R}_{2}, \ldots, \widehat{R}_{p}, \widehat{R}_{1}\right) .
\end{aligned}
$$

Then (1.1) and (4.1) can be expressed by

$$
X=A^{H} X(I+G X)^{-1} A+H
$$


and

$$
\widehat{R}=\tilde{X}-A^{H} \tilde{X}(I+G \tilde{X})^{-1} A-H,
$$

respectively. By simple matrix operations, we can get from (4.9) and (4.10) the perturbation equation [16, section 3$]$

$$
\begin{aligned}
& \Delta X-A^{H}(I+\tilde{X} G)^{-1} \Delta X(I+G \tilde{X})^{-1} A=\widehat{R} \\
& \quad+A^{H}(I+\tilde{X} G)^{-1} \Delta X(I+G \tilde{X})^{-1} G \Delta X\left[I+(I+G \tilde{X})^{-1} G \Delta X\right]^{-1}(I+G \tilde{X})^{-1} A,
\end{aligned}
$$

or equivalently,

$$
\mathbf{L}\left(\Delta X_{1}, \ldots, \Delta X_{p-1}, \Delta X_{p}\right)=\left(\widehat{R}_{2}, \ldots, \widehat{R}_{p}, \widehat{R}_{1}\right)+\left(f_{2}\left(\Delta X_{2}\right), \ldots, f_{p}\left(\Delta X_{p}\right), f_{1}\left(\Delta X_{1}\right)\right),
$$

where $\mathbf{L}$ is the linear operator defined by $(4.2), \widehat{R}_{j}(j=1, \ldots, p)$ are the residuals defined by (4.1), and the functions $f_{j}\left(\Delta X_{j}\right)(j=1, \ldots, p)$ are defined by

$$
\begin{aligned}
& f_{j}\left(\Delta X_{j}\right) \\
& =A_{j}^{H}\left(I+\tilde{X}_{j} G_{j}\right)^{-1} \Delta X_{j}\left(I+G_{j} \tilde{X}_{j}\right)^{-1} G_{j} \Delta X_{j}\left[I+\left(I+G_{j} \tilde{X}_{j}\right)^{-1} G_{j} \Delta X_{j}\right]^{-1} \\
& \quad \times\left(I+G_{j} \tilde{X}_{j}\right)^{-1} A_{j} .
\end{aligned}
$$

Since the matrix set $\left\{\tilde{\Phi}_{j}\right\}_{j=1}^{p}$ is pd-stable, the operator $\mathbf{L}$ is invertible. Consequently, the perturbation equation (4.12) can be expressed by

$$
\begin{aligned}
& \left(\Delta X_{1}, \ldots, \Delta X_{p-1}, \Delta X_{p}\right) \\
& \quad=\mathbf{L}^{-1}\left[\left(\widehat{R}_{2}, \ldots, \widehat{R}_{p}, \widehat{R}_{1}\right)+\left(f_{2}\left(\Delta X_{2}\right), \ldots, f_{p}\left(\Delta X_{p}\right), f_{1}\left(\Delta X_{1}\right)\right)\right] .
\end{aligned}
$$

Define the function $g\left(\Delta X_{1}, \ldots, \Delta X_{p-1}, \Delta X_{p}\right)$ on $\mathcal{H}_{n}^{p}$ by

$$
\begin{aligned}
& g\left(\Delta X_{1}, \ldots, \Delta X_{p-1}, \Delta X_{p}\right) \\
& \quad=\mathbf{L}^{-1}\left[\left(\widehat{R}_{2}, \ldots, \widehat{R}_{p}, \widehat{R}_{1}\right)+\left(f_{2}\left(\Delta X_{2}\right), \ldots, f_{p}\left(\Delta X_{p}\right), f_{1}\left(\Delta X_{1}\right)\right)\right] .
\end{aligned}
$$

Obviously, $g()$ can be regarded as a continuous mapping $\mathcal{M}: \mathcal{H}_{n}^{p} \rightarrow \mathcal{H}_{n}^{p}$, and the set of solutions to (4.14) is just the set of fixed points of the mapping $\mathcal{M}$.

Step 2. Estimates of some fixed points of $\mathcal{M}$.

From the definition (4.15) we get

$$
\left\|g\left(\Delta X_{1}, \ldots, \Delta X_{p}\right)\right\|_{F} \leq \epsilon+\frac{\left\|\left(f_{2}\left(\Delta X_{2}\right), \ldots, f_{p}\left(\Delta X_{p}\right), f_{1}\left(\Delta X_{1}\right)\right)\right\|_{F}}{l},
$$

where $\epsilon$ and $l$ are defined by (4.5). Moreover, from (4.13) we get

$$
\left\|f_{j}\left(\Delta X_{j}\right)\right\|_{F} \leq \frac{\phi_{j}^{2} \gamma_{j}\left\|\Delta X_{j}\right\|_{F}^{2}}{1-\gamma_{j}\left\|\Delta X_{j}\right\|_{F}^{2}} \leq \frac{\phi^{2} \gamma\left\|\Delta X_{j}\right\|_{F}^{2}}{1-\gamma\left\|\left(\Delta X_{1}, \ldots, \Delta X_{p}\right)\right\|_{F}}, \quad j=1, \ldots, p,
$$


where $\phi_{j}, \phi, \gamma_{j}, \gamma$ are defined by (4.4). Here we assume that the set $\left\{\Delta X_{j}\right\}_{j=1}^{p}$ satisfies

$$
1-\gamma\left\|\left(\Delta X_{1}, \ldots, \Delta X_{p}\right)\right\|_{F}>0 .
$$

Combining (4.16) and (4.17) gives

$$
\left\|g\left(\Delta X_{1}, \ldots, \Delta X_{p}\right)\right\|_{F} \leq \epsilon+\frac{\phi^{2} \gamma\left\|\left(\Delta X_{1}, \ldots, \Delta X_{p}\right)\right\|_{F}^{2}}{l\left(1-\gamma\left\|\left(\Delta X_{1}, \ldots, \Delta X_{p}\right)\right\|_{F}\right)} .
$$

By using the technique described by [16, section 4] we can prove that if $\epsilon$ satisfies the condition (4.6), then the mapping $\mathcal{M}$ has a fixed point $\left(\Delta X_{1}^{*}, \ldots, \Delta X_{p}^{*}\right)$ in the set

$$
\mathcal{S}_{r(\epsilon)}=\left\{\left(\Delta X_{1}, \ldots, \Delta X_{p}\right) \in \mathcal{H}_{n}^{p}:\left\|\left(\Delta X_{1}, \ldots, \Delta X_{p}\right)\right\|_{F} \leq r(\epsilon)\right\},
$$

where $r(\epsilon)$ is defined by (4.7).

Note that the condition (4.6) implies that for any $\left(\Delta X_{1}, \ldots, \Delta X_{p}\right) \in \mathcal{S}_{r(\epsilon)}$ the inequality (4.18) holds. In fact, if $\left(\Delta X_{1}, \ldots, \Delta X_{p}\right) \in \mathcal{S}_{r(\epsilon)}$, then we have

$$
\begin{aligned}
\gamma\left\|\left(\Delta X_{1}, \ldots, \Delta X_{p}\right)\right\|_{F} & \leq \gamma r(\epsilon) \quad(\text { by } \quad(4.20)) \\
& \leq \frac{2 \gamma \epsilon}{1+\gamma \epsilon} \quad(\text { by } \quad(4.7)) \\
& <\frac{l}{\phi^{2}+\phi \sqrt{\phi^{2}+l}+l} \quad(\text { by }(4.6)) \\
& \leq 1 .
\end{aligned}
$$

Step 3. On the matrix set $\left\{\tilde{X}_{j}-\Delta X_{j}^{*}\right\}_{j=1}^{p}$.

Let

$$
\Delta X^{*}=\operatorname{diag}\left(\Delta X_{1}^{*}, \ldots, \Delta X_{p}^{*}\right)
$$

and

$$
Y=\tilde{X}-\Delta X^{*}=\operatorname{diag}\left(Y_{1}, \ldots, Y_{p}\right) .
$$

Then from Step 1 we see that $Y$ is a Hermitian solution to the DARE (4.9); i.e., $Y$ satisfies

$$
Y=A^{H} Y(I+G Y)^{-1} A+H,
$$

or equivalently,

$$
\begin{aligned}
Y & -A^{H}(I+Y G)^{-1} Y(I+G Y)^{-1} A \\
& =H+A^{H}(I+Y G)^{-1} Y G Y(I+G Y)^{-1} A .
\end{aligned}
$$

Observe the following two facts:

1. The matrix on the right-hand side of (4.22) is Hermitian p.s.d.

2. The matrix $(I+G Y)^{-1} A$ can be written as

$$
(I+G Y)^{-1} A=\left[I-(I+G \tilde{X})^{-1} G \Delta X^{*}\right](I+G \tilde{X})^{-1} A,
$$


or equivalently,

$$
\operatorname{cyc}\left\{\left(I+G_{j} Y_{j}\right)^{-1} A_{j}\right\}_{j=1}^{p}=\operatorname{cyc}\left\{\left[I-\left(I+G_{j} \tilde{X}_{j}\right)^{-1} G_{j} \Delta X_{j}^{*}\right]^{-1} \tilde{\Phi}_{j}\right\}_{j=1}^{p},
$$

where the matrices $\tilde{\Phi}_{j}$ are defined by (4.3), and by the hypotheses the matrix set $\left\{\tilde{\Phi}_{j}\right\}_{j=1}^{p}$ is pd-stable. Moreover, for $j=1, \ldots, p$ we have

$$
\begin{aligned}
& \left\|\left(I+G_{j} \tilde{X}_{j}\right)^{-1} G_{j} \Delta X_{j}^{*}\right\|_{2} \\
& \leq\left\|\left(I+G_{j} \tilde{X}_{j}\right)^{-1} G_{j}\right\|_{2}\left\|\Delta X_{j}^{*}\right\|_{2} \\
& \leq \gamma_{j} r(\epsilon) \quad(\text { by }(4.4) \text { and }(4.20)) \\
& \left.\leq \frac{2 \gamma l \epsilon}{(1+\gamma \epsilon) l+\sqrt{(1+\gamma \epsilon)^{2} l^{2}-4\left(\phi^{2}+l\right) \gamma l \epsilon}} \quad \text { by (4.4) and }(4.7)\right) \\
& \leq \frac{2 \gamma \epsilon}{1+\gamma \epsilon} \\
& <\frac{l}{\phi^{2}+\phi \sqrt{\phi^{2}+l}+l} \quad(\text { by }(4.6)) .
\end{aligned}
$$

Consequently, by Lemma 2.4 , the matrix set $\left\{\left[I-\left(I+G_{j} \tilde{X}_{j}\right)^{-1} G_{j} \Delta X_{j}^{*}\right]^{-1} \tilde{\Phi}_{j}\right\}_{j=1}^{p}$ is pd-stable. By (4.23), the matrix set $\left\{\left(I+G_{j} Y_{j}\right)^{-1} A_{j}\right\}_{j=1}^{p}$ is pd-stable. Further, by Lemma 2.2, the matrix

$$
\operatorname{cyc}\left\{\left(I+G_{j} Y_{j}\right)^{-1} A_{j}\right\}_{j=1}^{p}=(I+G Y)^{-1} A
$$

is d-stable.

Hence, the Hermitian matrix $Y=\operatorname{diag}\left(Y_{1}, \ldots, Y_{p}\right)$, as a solution to (4.22), is positive semidefinite [3, Proposition 2.1]; and so the matrix $Y$, as a Hermitian solution to the DARE (4.21), is positive semidefinite and stabilizing. By the uniqueness of the stabilizing solution to the DARE (4.21) [8, Proposition 1], we have $Y=X=$ $\operatorname{diag}\left\{X_{j}\right\}_{j=1}^{p}$, the unique Hermitian p.s.d. stabilizing solution to the DARE (4.9). Thus, the matrix set $\left\{Y_{j}\right\}_{j=1}^{p}$ is just the unique Hermitian p.s.d. stabilizing solution set to the P-DARE (1.1).

Overall, we have proved the estimate

$$
\left\|\left(\tilde{X}_{1}-X_{1}, \ldots, \tilde{X}_{p}-X_{p}\right)\right\|_{F}=\left\|\left(\Delta X_{1}^{*}, \ldots, \Delta X_{p}^{*}\right)\right\|_{F} \leq r(\epsilon) .
$$

Note that the function $r(\epsilon)$ defined by (4.7) has the Taylor expansion at $\epsilon=0$ :

$$
r(\epsilon)=\epsilon+\frac{\gamma \phi^{2}}{l} \epsilon^{2}+O\left(\epsilon^{3}\right) \quad \text { as } \epsilon \rightarrow 0 .
$$

Consequently, for sufficiently small $\left\|\mathbf{L}^{-1}\left(\widehat{R}_{1}, \ldots, \widehat{R}_{p}\right)\right\|_{F}$, we have the first order estimate

$$
\left\|\left(\tilde{X}_{1}-X_{1}, \ldots, \tilde{X}_{p}-X_{p}\right)\right\|_{F} \lesssim \epsilon=\left\|\mathbf{L}^{-1}\left(\widehat{R}_{1}, \ldots, \widehat{R}_{p}\right)\right\|_{F}
$$


5. Numerical results. We now use a simple numerical example to illustrate our results of sections 3 and 4. All computations were performed using MATLAB, version 6.1. The relative machine precision is $2.22 \times 10^{-16}$.

Example 5.1. Consider the P-DARE (1.1) with $n=2, p=3$, and

$$
\begin{aligned}
& A_{1}=\left(\begin{array}{cc}
0 & 0 \\
10^{m} & 0
\end{array}\right), \quad A_{2}=\left(\begin{array}{ll}
0 & 1 \\
0 & 0
\end{array}\right), \quad A_{3}=\left(\begin{array}{ll}
0 & 0 \\
0 & 1
\end{array}\right), \\
& B_{1}=\left(\begin{array}{ll}
1 & 0 \\
0 & 1
\end{array}\right), \quad B_{2}=\left(\begin{array}{ll}
1 & 0 \\
0 & 1
\end{array}\right), \quad B_{3}=\left(\begin{array}{l}
1 \\
0
\end{array}\right), \\
& C_{1}=\left(\begin{array}{ll}
1 & 0 \\
0 & 1
\end{array}\right), \quad C_{2}=(1,0), \quad C_{3}=(0,0), \\
& R_{1}=\left(\begin{array}{ll}
1 & 0 \\
0 & 1
\end{array}\right), \quad R_{2}=\left(\begin{array}{ll}
1 & 0 \\
0 & 1
\end{array}\right), \quad R_{3}=1 .
\end{aligned}
$$

By $G_{j}=B_{j} R_{j}^{-1} B_{j}^{T}$ and $H_{j}=C_{j}^{T} C_{j}(j=1,2,3)$, we have

$$
\begin{aligned}
& G_{1}=\left(\begin{array}{ll}
1 & 0 \\
0 & 1
\end{array}\right), \quad G_{2}=\left(\begin{array}{cc}
1 & 0 \\
0 & 1
\end{array}\right), \quad G_{3}=\left(\begin{array}{ll}
1 & 0 \\
0 & 0
\end{array}\right), \\
& H_{1}=\left(\begin{array}{ll}
1 & 0 \\
0 & 1
\end{array}\right), \quad H_{2}=\left(\begin{array}{ll}
1 & 0 \\
0 & 0
\end{array}\right), \quad H_{3}=\left(\begin{array}{ll}
0 & 0 \\
0 & 0
\end{array}\right) \text {. }
\end{aligned}
$$

Thus, the corresponding P-DARE (1.1) can be written

$$
\left\{\begin{array}{l}
X_{3}=A_{1}^{T}\left(I+G_{1} X_{1}\right)^{-1} A_{1}+H_{1}, \\
X_{1}=A_{2}^{T}\left(I+G_{2} X_{2}\right)^{-1} A_{2}+H_{2}, \\
X_{2}=A_{3}^{T}\left(I+G_{3} X_{3}\right)^{-1} A_{3}+H_{3},
\end{array}\right.
$$

where $A_{j}$ and $G_{j}, H_{j}$ are the matrices of (5.1) and (5.2), respectively.

By (5.1), (2.14), and (2.15), we get the matrices $\mathcal{A}_{j}, \mathcal{B}_{j}$, and $\mathcal{C}_{j}(j=1,2,3)$ with

$$
\begin{aligned}
& \mathcal{A}_{1}=A_{3} A_{2} A_{1}=\left(\begin{array}{ll}
0 & 0 \\
0 & 0
\end{array}\right), \\
& \mathcal{B}_{1}=\left(A_{3} A_{2} B_{1}, A_{3} B_{2}, B_{3}\right)=\left(\begin{array}{ccccc}
0 & 0 & 0 & 0 & 1 \\
0 & 0 & 0 & 1 & 0
\end{array}\right), \\
& \mathcal{C}_{1}=\left(C_{1}^{T}, A_{1}^{T} C_{2}^{T}, A_{1}^{T} A_{2}^{T} C_{3}^{T}\right)^{T}=\left(\begin{array}{cccc}
1 & 0 & 0 & 0 \\
0 & 1 & 0 & 0
\end{array}\right)^{T}, \\
& \mathcal{A}_{2}=A_{2} A_{1} A_{3}=\left(\begin{array}{ll}
0 & 0 \\
0 & 0
\end{array}\right), \\
& \mathcal{B}_{2}=\left(A_{2} A_{1} B_{3}, A_{2} B_{1}, B_{2}\right)=\left(\begin{array}{ccccc}
10^{m} & 0 & 1 & 1 & 0 \\
0 & 0 & 0 & 0 & 1
\end{array}\right), \\
& \mathcal{C}_{2}=\left(C_{3}^{T}, A_{3}^{T} C_{1}^{T}, A_{3}^{T} A_{1}^{T} C_{2}^{T}\right)^{T}=\left(\begin{array}{cccc}
0 & 0 & 0 & 0 \\
1 & 0 & 1 & 0
\end{array}\right)^{T}, \\
& \mathcal{A}_{3}=A_{1} A_{3} A_{2}=\left(\begin{array}{ll}
0 & 0 \\
0 & 0
\end{array}\right), \\
& \mathcal{B}_{3}=\left(A_{1} A_{3} B_{2}, A_{1} B_{3}, B_{1}\right)=\left(\begin{array}{ccccc}
0 & 0 & 0 & 1 & 0 \\
0 & 0 & 10^{m} & 0 & 1
\end{array}\right) \text {, } \\
& \mathcal{C}_{3}=\left(C_{2}^{T}, A_{2}^{T} C_{3}^{T}, A_{2}^{T} A_{3}^{T} C_{1}^{T}\right)^{T}=\left(\begin{array}{cccc}
1 & 0 & 0 & 0 \\
0 & 0 & 0 & 0
\end{array}\right)^{T} \text {. }
\end{aligned}
$$


TABLE 5.1

Estimates of relative backward errors $(k=12)$.

\begin{tabular}{c|c|c|c}
\hline$m$ & $l^{*}$ & $u^{*}$ & $c_{\text {rel }}\left(X_{1}, X_{2}, X_{3}\right)$ \\
\hline 0 & $8.8 \times 10^{-13}$ & $8.8 \times 10^{-13}$ & 2.7 \\
\hline 1 & $5.7 \times 10^{-11}$ & $5.7 \times 10^{-11}$ & $7.1 \times 10$ \\
\hline 2 & $5.7 \times 10^{-9}$ & $5.7 \times 10^{-9}$ & $7.1 \times 10^{3}$ \\
\hline 3 & $3.5 \times 10^{-7}$ & $7.8 \times 10^{-7}$ & $7.1 \times 10^{5}$ \\
\hline 4 & $*$ & $*$ & $7.1 \times 10^{7}$ \\
\hline
\end{tabular}

It can be verified that the matrix pairs $\left(\mathcal{A}_{j}, \mathcal{B}_{j}\right)$ are d-stabilizable, and $\left(\mathcal{A}_{j}, \mathcal{C}_{j}\right)$ are d-detectable for $j=1,2,3$; i.e., the matrix pair sets $\left\{\left(A_{j}, B_{j}\right)\right\}_{j=1}^{3}$ and $\left\{\left(A_{j}, C_{j}\right)\right\}_{j=1}^{3}$ are pd-stabilizable and pd-detectable, respectively. By Theorem 2.5, the P-DARE (5.3) has a unique symmetric p.s.d. stabilizing solution set $\left\{X_{j}\right\}_{j=1}^{3}$. It is easy to verify that the set $\left\{X_{j}\right\}_{j=1}^{3}$ with

$$
X_{1}=\left(\begin{array}{cc}
1 & 0 \\
0 & 0
\end{array}\right), \quad X_{2}=\left(\begin{array}{cc}
0 & 0 \\
0 & 1
\end{array}\right), \quad X_{3}=\left(\begin{array}{ll}
1 & 0 \\
0 & 1
\end{array}\right)
$$

is the unique symmetric p.s.d. stabilizing solution set, which is independent of the values of $m$.

Let the approximate symmetric p.s.d. solution sets $\left\{\tilde{X}_{j}\right\}_{j=1}^{3}$ be given by

$$
\begin{aligned}
& \tilde{X}_{1}=X_{1}+\left(\begin{array}{rr}
-0.3 & -0.2 \\
-0.2 & 0.8
\end{array}\right) \times 10^{-k}, \quad \tilde{X}_{2}=X_{2}+\left(\begin{array}{rr}
0.1 & 0.1 \\
0.1 & -0.2
\end{array}\right) \times 10^{-k}, \\
& \tilde{X}_{3}=X_{3}+\left(\begin{array}{rr}
-0.2 & 0.3 \\
0.3 & 0.6
\end{array}\right) \times 10^{-k}, \quad k=0,1,2, \ldots
\end{aligned}
$$

We now are going to give estimates of backward errors and residual bounds for the approximate symmetric p.s.d. solution sets.

Estimates of backward errors. Some numerical results on backward errors of the approximate solution sets are listed in Tables 5.1 and 5.2 , where the bounds $l^{*}$ and $u^{*}$ are computed by (3.17)-(3.18), and the values of the relative condition number $c_{\text {rel }}\left(X_{1}, X_{2}, X_{3}\right)$ listed in Table 5.1 are computed by [12, (4.24)] with

$$
\xi_{j}=\left\|X_{j}\right\|_{F}, \quad \alpha_{j}=\left\|A_{j}\right\|_{F}, \quad \gamma_{j}=\left\|G_{j}\right\|_{F}, \quad \eta_{j}=\left\|H_{j}\right\|_{F}, \quad j=1,2,3 .
$$

The cases when the condition (3.15) of Theorem 3.1 is violated are denoted by asterisks in Tables 5.1 and 5.2.

From the results listed in Table 5.1 we see that the relative backward error increases as the relative conditioning of the P-DARE deteriorates.

The results listed in Tables 5.1 and 5.2 show that the relative backward errors are very small $\left(\eta\left(\left\{\tilde{X}_{j}\right\}_{j=1}^{3}\right) \lesssim 5.7 \times 10^{-9}\right)$ in the cases of $k=12$ and $m \leq 2$, and in the cases of $m=1$ and $k \geq 10$; this means that in such cases each approximate symmetric p.s.d. solution set $\left\{\tilde{X}_{j}\right\}_{j=1}^{3}$ is an exact symmetric p.s.d. solution set to a slightly perturbed P-DARE.

From the results listed in Table 5.2 we see that the relative backward error decreases as the error $\|\tilde{X}-X\|_{F}=\sqrt{\sum_{j=1}^{3}\left\|\tilde{X}_{j}-X_{j}\right\|_{F}^{2}}$ decreases. 
TABLE 5.2

Estimates of relative backward errors $\left(m=1, \quad c_{\text {rel }}\left(X_{1}, X_{2}, X_{3}\right) \approx 71\right)$.

\begin{tabular}{c|c|c|c}
\hline$k$ & $l^{*}$ & $u^{*}$ & $\|\tilde{X}-X\|_{F}$ \\
\hline 2 & $*$ & $*$ & $1.2 \times 10^{-2}$ \\
\hline 4 & $3.4 \times 10^{-3}$ & $7.9 \times 10^{-3}$ & $1.2 \times 10^{-4}$ \\
\hline 6 & $5.7 \times 10^{-5}$ & $5.7 \times 10^{-5}$ & $1.2 \times 10^{-6}$ \\
\hline 8 & $5.7 \times 10^{-7}$ & $5.7 \times 10^{-7}$ & $1.2 \times 10^{-8}$ \\
\hline 10 & $5.7 \times 10^{-9}$ & $5.7 \times 10^{-9}$ & $1.2 \times 10^{-10}$ \\
\hline 12 & $5.7 \times 10^{-11}$ & $5.7 \times 10^{-11}$ & $1.2 \times 10^{-12}$ \\
\hline
\end{tabular}

Computed results for this example show that

$$
l_{j} \approx u_{j} \approx \rho_{j}, \quad j=1,2,3,
$$

which mean that the linear estimates (3.19) are relatively sharp, while the nonlinear estimates (3.16) do not even exist in some cases. However, it is worth pointing out that the nonlinear estimates (3.16) guarantee the existence of the solution to the optimization problem (3.5), while the linear estimates (3.19) would formally give approximate bounds which might not correspond to any solution to the problem $(3.5)$.

Residual bounds. Here we only present a few results in the case of $m=0$. In such a case, the relative condition number $c_{\text {rel }}\left(X_{1}, X_{2}, X_{3}\right) \approx 2.7$. Taking $k=4$ in (5.4) we obtain an approximate symmetric p.s.d. solution set $\left\{\tilde{X}_{j}\right\}_{j=1}^{3}$, among which each $\tilde{X}_{j}$ approximates $X_{j}(1 \leq j \leq 3)$ up to 5 significant figures.

A computation by (4.7) gives

$$
\frac{r(\epsilon)}{\left\|\tilde{X}_{1}\right\|_{F}} \approx 1.5 \times 10^{-4}, \quad \frac{r(\epsilon)}{\left\|\tilde{X}_{2}\right\|_{F}} \approx 1.5 \times 10^{-4}, \quad \frac{r(\epsilon)}{\left\|\tilde{X}_{3}\right\|_{F}} \approx 1.1 \times 10^{-4} .
$$

Combining the estimates with (4.8) we get relative error bounds for $\tilde{X}_{j}(j=1,2,3)$ :

$$
b_{\text {rel }}\left(\tilde{X}_{1}\right) \approx 1.5 \times 10^{-4}, \quad b_{\text {rel }}\left(\tilde{X}_{2}\right) \approx 1.5 \times 10^{-4}, \quad b_{\text {rel }}\left(\tilde{X}_{3}\right) \approx 1.1 \times 10^{-4} .
$$

From (5.5) we see that the approximate symmetric p.s.d. solution set $\left\{\tilde{X}_{j}\right\}_{j=1}^{3}$ has at least 4 correct digits.

Note that by Theorem 4.1 the estimate (4.7) can only be applied to the case where the condition (4.6) is satisfied; i.e.,

$$
\delta(\epsilon) \equiv \frac{l}{\gamma\left(2 \phi^{2}+2 \phi \sqrt{\phi^{2}+l}+l\right)}-\epsilon>0 .
$$

The results listed in Table 5.3 show the scope of application of the estimate (4.7) for this example.

Example 5.2 (see [12, Example 5.1]). Consider the P-DARE (1.1) with $n=3, p=$ 3 , and

$$
A_{j}=V_{j}^{T} A_{j}^{(0)} V_{j}, \quad G_{j}=V_{j}^{T} G_{j}^{(0)} V_{j}, \quad H_{j}=V_{j}^{T} H_{j}^{(0)} V_{j}, \quad j=1,2,3,
$$


TABLE 5.3

\begin{tabular}{c|c|c}
\hline$m$ & $c_{\text {rel }}\left(X_{1}, X_{2}, X_{3}\right)$ & $\delta(\epsilon)$ \\
\hline 0 & 2.7 & $\delta(\epsilon)>0$ if and only if $k \geq 1$ \\
\hline 1 & $7.1 \times 10$ & $\delta(\epsilon)>0$ if and only if $k \geq 11$ \\
\hline 2 & $7.1 \times 10^{3}$ & $\delta(\epsilon)>0$ if and only if $k \geq 13$ \\
\hline 3 & $7.1 \times 10^{5}$ & $\delta(\epsilon)>0$ if and only if $k \geq 21$ \\
\hline
\end{tabular}

TABLE 5.4

Estimates of relative backward errors.

\begin{tabular}{c|c|c}
\hline$m$ & $l^{*}$ & $u^{*}$ \\
\hline 0 & $2.2 \times 10^{-15}$ & $2.2 \times 10^{-15}$ \\
\hline 1 & $1.9 \times 10^{-15}$ & $1.9 \times 10^{-15}$ \\
\hline 2 & $3.8 \times 10^{-15}$ & $3.8 \times 10^{-15}$ \\
\hline 3 & $1.9 \times 10^{-14}$ & $1.9 \times 10^{-14}$ \\
\hline 4 & $4.0 \times 10^{-13}$ & $4.0 \times 10^{-13}$ \\
\hline 5 & $5.0 \times 10^{-12}$ & $5.0 \times 10^{-12}$ \\
\hline 6 & $5.0 \times 10^{-11}$ & $5.0 \times 10^{-11}$ \\
\hline
\end{tabular}

where

$$
\begin{aligned}
& A_{1}^{(0)}=\operatorname{diag}\left(0,10^{-m}, 1\right), \quad A_{2}^{(0)}=\operatorname{diag}\left(10^{-9}, 10^{-m}, 1+10^{-3}\right), \\
& A_{3}^{(0)}=\operatorname{diag}\left(10^{-3}, 10^{-m+1}, 0.5\right), \\
& G_{j}^{(0)}=\operatorname{diag}\left(\frac{1}{j} 10^{-m}, \frac{1}{j} 10^{-m}, j \times 10^{-m}\right), \quad H_{j}^{(0)}=\operatorname{diag}\left(\frac{1}{j} 10^{m}, j, j \times 10^{-m}\right),
\end{aligned}
$$$$
j=1,2,3,
$$

and

$$
\begin{aligned}
& V_{1}=I-2 v_{1} v_{1}^{T} \quad \text { with } \quad v_{1}=\frac{1}{\sqrt{3}}(1,1,1)^{T}, \\
& V_{2}=I-2 v_{2} v_{2}^{T} \quad \text { with } \quad v_{2}=\frac{1}{\sqrt{6}}(1,1,2)^{T}, \\
& V_{3}=I-2 v_{3} v_{3}^{T} \quad \text { with } \quad v_{3}=\frac{1}{\sqrt{11}}(-1,1,3)^{T} .
\end{aligned}
$$

By applying the file "dare" of Control System Toolbox, we get computed symmetric p.s.d. solution sets $\left\{\tilde{X}_{1}, \tilde{X}_{2}, \tilde{X}_{3}\right\}$ to the P-DARE (1.1). Some numerical results on backward errors and residual bounds for the computed solution sets are listed in Tables 5.4 and 5.5 , respectively, where the relative error bounds $b_{\text {rel }}\left(\tilde{X}_{j}\right)$ for $\tilde{X}_{j}(j=1,2,3)$ are defined by (4.8).

The results listed in Table 5.4 show that each computed symmetric p.s.d. solution set $\left\{\tilde{X}_{1}, \tilde{X}_{2}, \tilde{X}_{3}\right\}$ by applying the file "dare" of Control System Toolbox is the exact symmetric p.s.d. solution set to a slightly perturbed P-DARE; in other words, the computation has proceeded stably.

From the results listed in Table 5.5 we see that the computed symmetric p.s.d. solution sets $\left\{\tilde{X}_{1}, \tilde{X}_{2}, \tilde{X}_{3}\right\}$ have high relative precision when $m$ is a small natural number; e.g., in the case $m=3$, each computed $\tilde{X}_{j}$ has at least 14 correct digits. 
TABLE 5.5

Residual bounds.

\begin{tabular}{c|c|c|c|c|c}
\hline$m$ & $\epsilon$ & $r(\epsilon)$ & $b_{\text {rel }}\left(\tilde{X}_{1}\right)$ & $b_{\text {rel }}\left(\tilde{X}_{2}\right)$ & $b_{\text {rel }}\left(\tilde{X}_{3}\right)$ \\
\hline 0 & $8.3 \times 10^{-13}$ & $8.3 \times 10^{-13}$ & $2.1 \times 10^{-13}$ & $7.5 \times 10^{-15}$ & $3.2 \times 10^{-13}$ \\
\hline 1 & $1.3 \times 10^{-14}$ & $1.3 \times 10^{-14}$ & $2.3 \times 10^{-15}$ & $2.3 \times 10^{-15}$ & $1.3 \times 10^{-15}$ \\
\hline 2 & $3.3 \times 10^{-13}$ & $3.3 \times 10^{-13}$ & $6.2 \times 10^{-15}$ & $9.6 \times 10^{-15}$ & $3.2 \times 10^{-15}$ \\
\hline 3 & $2.0 \times 10^{-11}$ & $2.0 \times 10^{-11}$ & $3.8 \times 10^{-14}$ & $6.0 \times 10^{-14}$ & $2.0 \times 10^{-14}$ \\
\hline 4 & $4.2 \times 10^{-9}$ & $4.2 \times 10^{-9}$ & $8.0 \times 10^{-13}$ & $1.2 \times 10^{-12}$ & $4.1 \times 10^{-13}$ \\
\hline 5 & $5.4 \times 10^{-7}$ & $5.4 \times 10^{-7}$ & $1.0 \times 10^{-11}$ & $1.6 \times 10^{-11}$ & $5.3 \times 10^{-12}$ \\
\hline 6 & $5.3 \times 10^{-5}$ & $5.3 \times 10^{-5}$ & $1.0 \times 10^{-10}$ & $1.6 \times 10^{-10}$ & $5.3 \times 10^{-11}$ \\
\hline
\end{tabular}

Acknowledgment. I would like to thank Wen-Wei Lin, who gave me the computed symmetric p.s.d. solution sets $\left\{\tilde{X}_{1}, \tilde{X}_{2}, \tilde{X}_{3}\right\}$ to the P-DARE (1.1) of Example 5.2 by applying the file "dare" of Control System Toolbox. I also thank the referees for helpful comments and suggestions.

\section{REFERENCES}

[1] S. Bittanti, P. Colaneri, and G. De Nicolao, The difference periodic Riccati equation for the periodic prediction problem, IEEE Trans. Automat. Control, 33 (1988), pp. 706-712.

[2] S. Bittanti, P. Colaneri, and G. De Nicolao, The periodic Riccati equation, in The Riccati Equations, S. Bittanti, A. Laub, and J. Willems, eds., Springer-Verlag, Berlin, 1991.

[3] P. M. Gahinet, A. J. Laub, C. S. Kenney, and G. A. Hewer, Sensitivity of the stable discrete-time Lyapunov equation, IEEE Trans. Automat. Control, 35 (1990), pp. 12091217.

[4] A. R. Ghavimi And A. J. Laub, Backward error, sensitivity, and refinement of computed solutions of algebraic Riccati equations, Numer. Linear Algebra Appl., 2 (1995), pp. 29-49.

[5] A. Graham, Kronecker Products and Matrix Calculus: With Applications, John Wiley, New York, 1981.

[6] N. J. Higham, Perturbation theory and backward error for $A X-X B=C$, BIT, 33 (1993), pp. $124-136$.

[7] N. J. Higham, Accuracy and Stability of Numerical Algorithms, 2nd ed., SIAM, Philadelphia, 2002.

[8] V. IONESCU AND M. Weiss, On computing the stabilizing solution of the discrete-time Riccati equation, Linear Algebra Appl., 174 (1992), pp. 229-238.

[9] B. KÅgströM, A perturbation analysis of the generalized Sylvester equation $(A R-L B, D R-$ $L E)=(C, F)$, SIAM J. Matrix Anal. Appl., 15 (1994), pp. 1045-1060.

[10] M. M. Konstantinov and P. Hr. Petkov, Note on "Perturbation theory for algebraic Riccati equations," SIAM J. Matrix Anal. Appl., 21 (1999), p. 327.

[11] M. M. Konstantinov, P. Hr. Petkov, V. Mehrmann, and D. Gu, Additive matrix operators, in Proceedings of the 30th Spring Conference of Bulgarian Mathematicians, Borovets (Bulgaria), 2001, pp. 169-175.

[12] W.-W. Lin AND J.-G. Sun, Perturbation analysis of the periodic discrete-time algebraic Riccati equation, SIAM J. Matrix Anal. Appl., 24 (2002), pp. 411-438.

[13] G. W. Stewart and J.-G. Sun, Matrix Perturbation Theory, Academic Press, Boston, 1990.

[14] J.-G. Sun, Residual bounds of approximate solutions of the algebraic Riccati equation, Numer. Math., 76 (1997), pp. 249-263.

[15] J.-G. Sun, Backward error for the discrete-time algebraic Riccati equation, Linear Algebra Appl., 259 (1997), pp. 183-208.

[16] J.-G. Sun, Residual bounds of approximate solutions of the discrete-time algebraic Riccati equation, Numer. Math., 78 (1998), pp. 463-478.

[17] J.-G. Sun, Stability and Accuracy: Perturbation Analysis of Algebraic Eigenproblems, Report UMINF 98.07 (revised), ISSN-0348-0542, Department of Computing Science, Umeå University, 2002. 